\title{
High-Frequency Sections of Active Filters of Mixed-Signal SoC Based on Current Amplifiers
}

\author{
Sergei Krutchinsky and Nikolai Prokopenko \\ South Russia State University of Economics and Service, Russia \\ Correspondence should be addressed to Nikolai Prokopenko, prokopenko@sssu.ru \\ Received 10 July 2012; Accepted 28 August 2012 \\ Academic Editors: H. A. Alzaher, M. Hopkinson, and R. Senani
}

Copyright ( $) 2012$ S. Krutchinsky and N. Prokopenko. This is an open access article distributed under the Creative Commons Attribution License, which permits unrestricted use, distribution, and reproduction in any medium, provided the original work is properly cited.

The sufficient conditions for the efficient use of active elements are formulated by analyzing the basic structure of second-order unit. The expediency of current amplifiers usage in HF and SHF filters is shown. The examples of a methodical nature are given and conclusions of application importance are formulated.

\section{Introduction}

A modern mixed-signal systems on a chip (SoC) creating for communications and radio-technical systems are inextricably linked to the development of analog and analogdigital IP-blocks for a sufficiently accurate processing of HF and SHF range signals. Some of the basic components of these blocks are frequency filters, which are highly sensitive parametrically. As a rule, reduction of the parametric sensitivity causes the need to create fairly complex active elements, which consume much power. Exactly energy problems of precision filters reduce the attainable level of integration of the SoC and the area of practical usage. Typically, the frequency filter is designed for a specific task with given parametric and structural constraints that are defined by a specific technology. In HF and SHF ranges transimpedance amplifiers (voltage-current converters) are often used as the active elements for compensation of losses in the passive RCcircuits $[1,2]$. However, the potentially achievable unity-gain frequencies of these elements give way to current amplifiers, thus ultimately limiting the product of the $Q$ factor $(Q)$ and the pole frequency $\left(f_{p}\right)$ with the existing restrictions on power consumption. That is why the potential properties of the filter based on current amplifiers have a practical perspective and require further theoretical research. At least, the limiting theoretical estimations are not yet available and practical recommendations are not formulated.

\section{Problem Statement}

As a rule, to increase the stability of the frequency characteristics of high-order filters, additional intersection connections are used to ensure the minimization of sensitivity to the instability of the passive $\mathrm{RC}$ elements in the range of operating frequencies [3, 4]. In HF and SHF bands, this schematic approach is almost never used because of the high and usually not accessible requirements to a parasitic phase shifts of used active elements. In this regard, the cascading of the second-order sections remains the main direction of the implementation of the necessary poles and zeros of the desired transfer function $[5,6]$. That is why improving of their parameters stability and reduction (minimization) of appropriate parametric sensitivities is the main way of the general problem solution. In addition, achievable through the implementation in modern technologies the precision of resistors and capacitors in frequency-defining elements is not sufficient to ensure admissible errors of transfer function poles of the main frequency filter types.

According to-above-mentioned things, solutions are necessary for the whole class of interrelated circuitry tasks which are aimed on the accuracy improving of the basic parameters realization in the second-order sections of HF and SHF ranges.

Firstly, taking into account the dominating influence of the pole frequency implementation error $\left(\delta f_{p}\right)$ on the 
capacitive characteristics of high- $Q$ sections, it is necessary to create structures with a minimum parametric sensitivity to the instability of active elements and components, and first of all, current amplifiers [7]. Secondly, from the point of view of frequency-band extension, similar structures with maximally effective usage of these amplifiers resources (component and energy costs for the product of Q-factor and pole frequency) are needed [8]. At the same time possible and acceptable trade-off is the equality of contributions of attenuation instability $\left(\delta d_{p}=-\delta Q\right)$ and pole frequency instability $-\delta f_{p} Q \approx \delta Q$. In terms of general problem solving it creates sufficient preconditions for energy consumption reduction or provides the structural degrees of freedom to increase the operating frequency range. Finally, and this is the most important from a technological point of view, the existing structural and hence the parametric degrees of freedom can be used for correction (adjustment) of the pole parameters $\left(f_{p}, Q\right)$ by mode-defining and therefore low-frequency circuits. Specified property of such circuit solutions permits not only to integrate IP-blocks and SoC, but also to implement during their exploitation a temperature and temporal correction of plural transformation tracts through programmable cores of the system and simple digital-to-analog converters.

\section{The Main Properties of the Basic Structure of the Second-Order Section}

In general case the structure (Figure 1) consists of three autonomous units- the active element $K$, the frequencydependent $\gamma(p)$, and frequency-independent $\beta$ circuits.

That is why its transfer function

$$
F(p)=\frac{K(p)\left[\gamma_{1}(p)-\beta_{1}\right]}{1-K(p)\left[\gamma_{2}(p)-\beta_{2}\right]}, \quad\left(K(p)=\frac{K}{1+p \tau_{a}}\right)
$$

allows to realize any type of filter, amplitude, or phase corrector. In general case for the second-order section

$$
\begin{gathered}
\gamma_{1}(p)=\frac{N(p)}{p^{2}+p D_{p} \omega_{p c}+\omega_{p c}^{2}}, \\
\gamma_{2}(p)=\gamma_{0} \frac{a_{2} p^{2}+a_{1} p D_{0} \omega_{p c}+a_{0} \omega_{p c}^{2}}{p^{2}+p D_{p} \omega_{p c}+\omega_{p c}^{2}},
\end{gathered}
$$

where $D_{0}, D_{p}$ are the attenuation of zero and the pole of frequency-dependent circuit; $\omega_{p c}$ is the frequency of its pole; $\gamma_{n}$ is the scale transfer coefficient; $a_{i}$ is binary $(0,1)$ coefficients; $N(p)$ is the polynomial that defines the type of implemented second-order section.

From (1)-(3) follows that for function in general form

$$
F(p)=\frac{A(p)}{p^{2}+p d_{p} \omega_{p}+\omega_{p}^{2}}
$$

frequency $\omega_{p}$ and pole attenuation $d_{p}$ for idealized schema $\left(\tau_{a}=0\right)$

$$
\begin{gathered}
\omega_{(p)}=\omega_{p c} \sqrt{\frac{1+\beta_{2} K-K \gamma_{0} a_{0}}{1+\beta_{2} K-K \gamma_{0} a_{2}}}, \\
d_{p}=\frac{D_{p}\left(1+\beta_{2} K\right)-K \gamma_{0} D_{0} a_{1}}{\sqrt{\left(1+K \beta_{2}-K \gamma_{0} a_{2}\right)\left(1+K \beta_{2}-K \gamma_{0} a_{0}\right)}} .
\end{gathered}
$$

Ratios (5) and (6) define structure of parameters influence of passive frequency-dependent $\left(D_{0}, D_{p}, \omega_{p c}, \gamma_{0}\right)$, frequency-independent $\left(\beta_{2}\right)$ circuits, and active $(K)$ elements and allow to receive the following practical conclusions. First, for exception of the most unstable parameter influence of the scheme $K$ on frequency of a pole $\omega_{p}$, it is necessary to use symmetric frequency-defining circuits $\left(a_{2}=a_{0}\right)$ when $\omega_{p}=$ $\omega_{p c}$ and active element influence is directed on increase of $Q$-factor $Q=1 / d_{p}$. Secondly, in case of use of noninverting amplifier or the converter $(K>0)$ value $d_{p}(6)$ is minimized at $a_{0}=a_{2}=0, a_{1}=1$

$$
d_{p}=D_{p}-\gamma_{0} D_{0} \frac{K}{1+K \beta_{2}},
$$

and at application of an inverting active element $(K<0)$ this minimization is possible when $a_{0}=a_{1}=a_{2}=1, \beta_{2}=0$ :

$$
d_{p}=D_{0} \frac{K \gamma_{0}}{1+K \gamma_{0}}+\frac{D_{p}}{1+K \gamma_{0}} .
$$

Thus, in the first case $(K>0)$ in feedback loop of an active element (the amplifier and the converter) $K$ it is necessary to use the frequency-defining circuits of band-pass type, and in the second case $(K<0)$-rejection type. In this case frequency of a pole of the scheme will be always defined by frequency of passive circuit pole, and action of active element will be directed on increase of realized $Q$ factor.

For passive RC circuits of the second order [5] of bandpass type the condition $D_{0} \gamma_{0}=D_{p}-D_{0}$ is characteristic, so at $\beta_{2}=0$

$$
d_{p}=D_{p}(1-K)+D_{0} K .
$$

Therefore, by the choice of passive elements ratios of these circuits (e.g., T-bridges) and structure of the amplifier $K$ it is possible to provide realization of $\omega_{p}$, and $d_{p}$ with low parametrical sensitivity. Apparently from (8), realization of this condition for inverting amplifiers is connected with limiting $(K \rightarrow \infty)$ increase of its gain.

As it is seen from (1), (2), the execution of the formulated conditions demands from passive frequency-defining circuits the additional condition realization of reproduction of the desired frequency characteristics in a circuit of direct transfer $\left(\gamma_{1}(p)\right)$.

If to consider the influence of time constant of noninverting active elements $\left(\tau_{a}\right)$ on parameters of transfer function poles of basic structure then under condition of their low admissible relative changes $\left(\delta \omega_{p}, \delta d_{p}\right)$ we receive

$$
\delta \omega_{p} \approx \delta_{d_{p}} \approx-\frac{1}{2} \frac{\omega_{p}}{B_{S}} D_{p} \frac{K}{1+\beta_{2} K},
$$




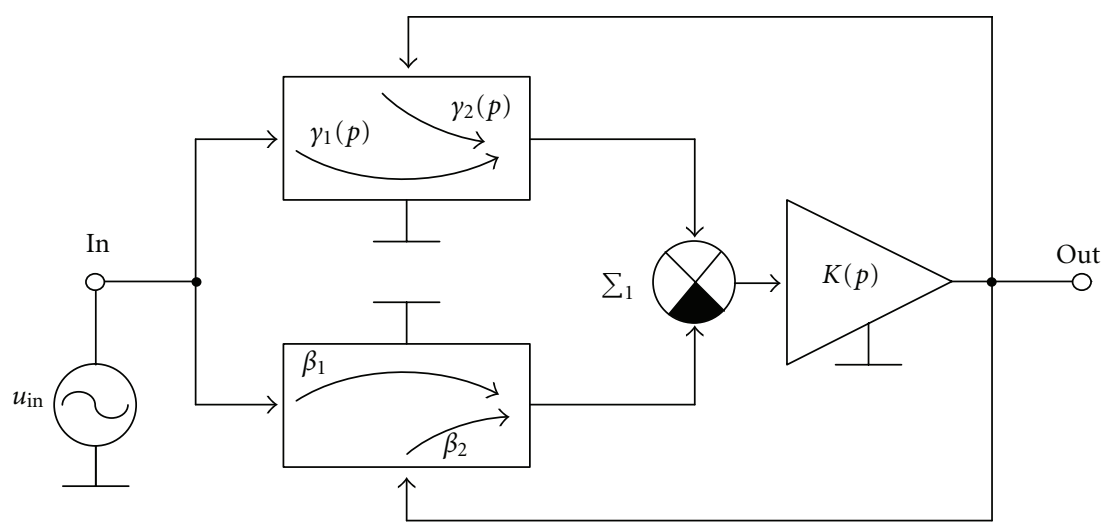

FIGURE 1: Basic structure of second-order section.

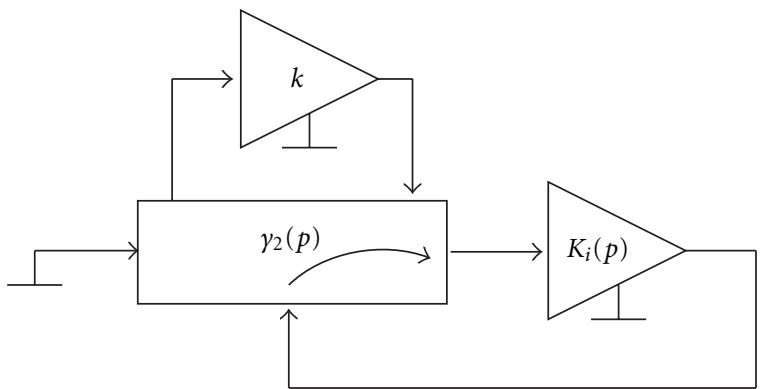

FIgURE 2: The feature of formation of SHF section poles.

where $B_{S}=K / \tau_{a}$ is the gain area of active element $K$ of basic structure.

In this case parametrical sensitivities

$$
S_{B_{S}}^{\omega_{p}}=S_{B_{S}}^{d_{p}}=\frac{1}{2} \frac{\omega_{p}}{B_{S}} D_{p} \frac{K}{1+\beta_{2} K}
$$

are defined by numerical values of $B_{S}$ and pole attenuation $D_{p}$ of a passive chain and can not be minimized by frequency-independent feedback depth $(0 \leq \beta \leq 1)$.

From the specified positions the most important qualitative parameter of passive frequency-defining circuits is their attenuation $D_{p}$ which reduction favorably effects on a working frequency range and realized $Q$-factor. For RC circuits of the second order [5] $D_{p \min }=3$, thus $D_{0}=2$, and under condition of $D_{0}$ minimization (see (13)) $D_{p}=2 Q$ and, therefore, the influence of $B_{S}$ on poles parameters increases. For reduction of $D_{p}$ it is possible to insert into RC circuit the additional repeater $k$ (Figure 2 ) that allows, in particular, to realize in schemes the additional useful structural and parametrical degrees of freedom.

\section{Low-Sensitive SHF Sections of Band Type}

The created above conclusions show that for active elements influence reduction on the frequency of high-Q pole it is expedient to use current repeaters $\left(K_{i}\right)$ in which feedback loop the frequency-dependent circuit of band-pass type with the additional voltage buffer repeater $k$ separating this circuit into elementary differentiating (integrating) circuits

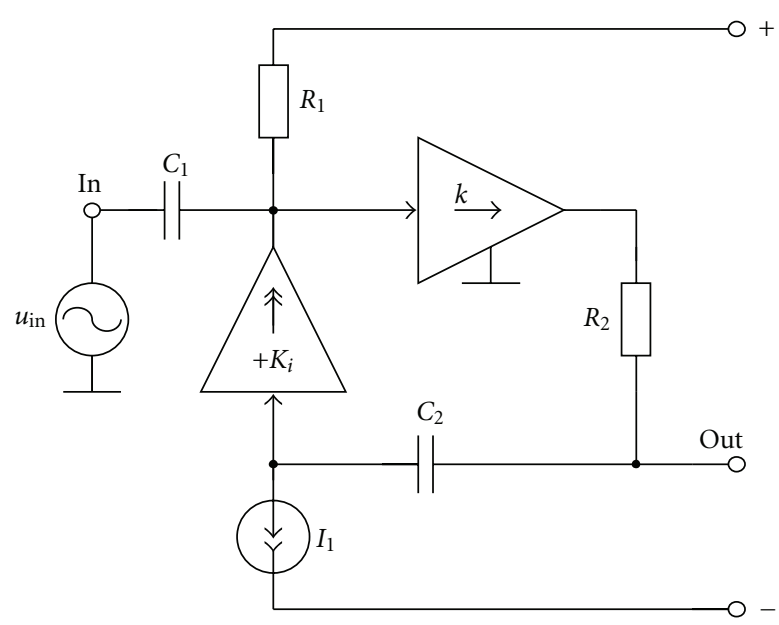

Figure 3: The structure of low-sensitive section of the band type filter.

is included. In this case for increasing of $B_{S}$ and, therefore, according to ratios $(10)$ and $(11)\left(\beta_{2}=0\right)$, the reduction of sensitivity and parameters realization errors, the circuitry degrees of freedom are created or, at least, saved, and $k$ usage allows to reduce attenuation of a passive circuit pole $D_{p}$. The structure of such section is shown on Figure 3.

Using a standard notation

$$
F(p)=K_{0} \frac{p d_{p} \omega_{p}}{p^{2}+p d_{p} \omega_{p}+\omega_{p}^{2}},
$$

it is easy to determine that

$$
\begin{gathered}
\omega_{p}=\frac{1}{\sqrt{\tau_{1} \tau_{2}}} ; \quad\left(\tau_{1}=R_{1} C_{1}, \tau_{2}=C_{2}\left(R_{2}+r_{i}+r_{u}\right)\right), \\
d_{p}=\sqrt{\frac{\tau_{1}}{\tau_{2}}}+\sqrt{\frac{\tau_{2}}{\tau_{1}}}\left(1-K_{i} \frac{R_{1}}{R_{2}+r_{i}+r_{u}}\right), \\
K_{0}=Q \sqrt{\frac{\tau_{1}}{\tau_{2}}},
\end{gathered}
$$

where $r_{i}, r_{u}$ are input resistance of current amplifier and output resistance of the buffer voltage amplifier $(k=1)$. 
Second-order circuit separation by means of the buffer $k$ allows to get

$$
D_{0}=\sqrt{\frac{\tau_{1}}{\tau_{2}}}, \quad D_{p}=\sqrt{\frac{\tau_{2}}{\tau_{1}}}+\sqrt{\frac{\tau_{1}}{\tau_{2}}},
$$

therefore parametrical sensitivities of the scheme can be minimized.

From (13) follows that always

$$
\begin{array}{rlrl}
S_{R_{1}}^{\omega_{p}} & =S_{C_{1}}^{\omega_{p}}=S_{C_{2}}^{\omega_{p}}=-\frac{1}{2}, & S_{R_{2}}^{\omega_{p}}=-\frac{1}{2} \frac{R_{2}}{R_{2}+r_{i}+r_{u}}, \\
S_{r_{i}}^{\omega_{p}}=-\frac{1}{2} \frac{r_{i}}{R_{2}+r_{i}+r_{u}}, & S_{r_{u}}^{\omega_{p}}=-\frac{1}{2} \frac{r_{u}}{R_{2}+r_{i}+r_{u}} .
\end{array}
$$

In addition, despite the differential realization principle of low pole attenuation (14) the minimization of its parametrical sensitivity to the changing of passive elements of discussed structure is possible. So, at execution of parametrical conditions

$$
R_{1}=R_{2}+r_{i}+r_{u}, \quad \tau_{1}=\tau_{2}\left(1-K_{i}\right)
$$

realized attenuation

$$
d_{p}=2 \sqrt{1-K_{i}}
$$

is accompanied by extremely low sensitivity

$$
S_{C_{1}}^{d_{p}}=S_{C_{2}}^{d_{p}}=S_{R_{1}}^{d_{p}}=S_{R_{2}+r_{i}+r_{u}}^{d_{p}}=0 .
$$

Thus, to ensure high qualitative indexes of schematics of band-pass sections it is necessary to develop special current amplifiers with transfer coefficient numerical values that following from (19). In this case, as it is evident from (15), the gain

$$
K_{0}=\frac{1}{2}
$$

is enough small and by multistage filter implementation with additional intersection connections can significantly affect the realizable dynamic range.

By such filter implementation it is essential according to (14), (15) to increase $K_{i}$ and to optimize the ratio of resistive elements of the scheme. In the general case

$$
K_{1} \frac{R_{1}}{R_{2}+r_{1}+r_{u}}=\frac{K_{0}\left(K_{0}-1\right)}{Q^{2}}+1
$$

As seen from the given ratio the required values of $K_{0}$ and $Q$ can be realized not only by the special structure of the current amplifier but also by selecting of the ratio of resistive elements $R_{1}$ and $R_{2}$. Particularly, this ratio can be used for designated parameters correction. In some cases the emerging degree of freedom can be used to optimize the sensitivity of the pole attenuation. Thus, to implement $K_{0}=Q$ it is needed to meet the conditions

$$
\tau_{1}=\tau_{2}, \quad K_{i} \frac{R_{1}}{R_{2}+r_{i}+r_{u}}=2-d p
$$

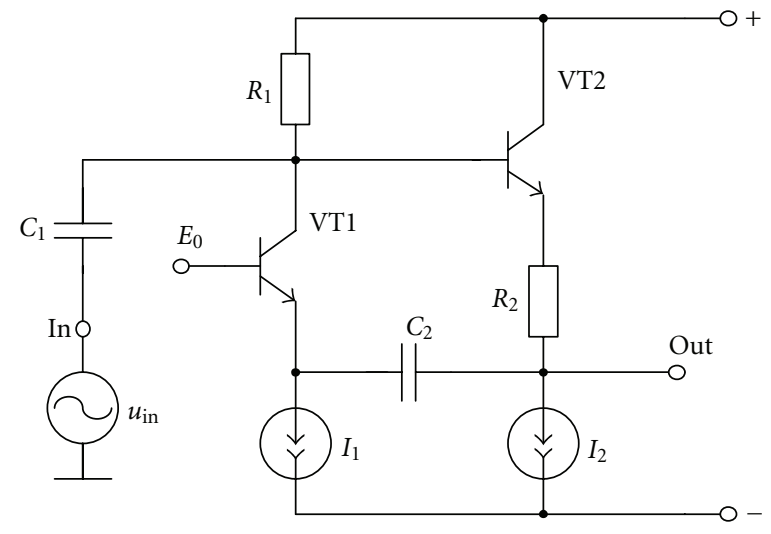

FIGURE 4: The simplest low-sensitivity section schematic of the band-pass filter.

in this case the parametric sensitivity

$$
S_{C_{1}}^{d p}=S_{R_{1}}^{d p}=-S_{C_{1}}^{d p}=-S_{R_{2}+r_{i}+r_{u}}^{d p}=Q-\frac{1}{2}
$$

is practically defined by realizable $Q$-factor.

When designing the multisection filters with additional intersection connection the required value of $K_{0}$ is limited to values that are close to unity [3]. In this case, for sensitivity reduction it is advisable to use conditions

$$
\tau_{1}=\tau_{2} d_{p}^{2}, \quad K_{i} R_{1}=R_{2}+r_{i}+r_{u},
$$

that ensure the equality of this sensitivity to unity.

\section{Examples of Second-Order Band-Pass Sections}

The simplest current amplifier $\left(K_{i}=\alpha\right)$ and repeater $(k)$ are the stages with common base and common collector that have the maximum $B_{s}$. Therefore, the simplest scheme of band-pass section is the scheme in Figure 4. Here

$$
K_{i}=\alpha_{1}, \quad r_{i}=h_{11.1}, \quad r_{u}=h_{11.2},
$$

where $h_{11 . i} \approx \varphi_{T} / I_{i}, \alpha_{i}$ are input resistance and static gain of emitter current of transistor with $i$ index.

According to (13) in this scheme the pole frequency adjustment is possible by changing of bipolar transistors operation modes (by sources $I_{1}$ and $I_{2}$ ). However, due to the influence of these parameters (expressions (14), (15)) on the pole attenuation $d_{p}$ and gain $K_{0}$, it is feasible only for small $Q=1 / d p$ values, when conditions (19) and (20) are satisfied. Otherwise, as is evident from modeling results of this scheme (Table 1), this leads to significant changes of specified parameters.

To eliminate this effect in the scheme it is necessary to use a more complex current amplifier that eliminates restrictions on the ratios of resistive elements of the scheme (expressions (22), (23), (25)) and allows to change gain $K_{i}$ regardless of the resistors $r_{i}$ and $r_{u}$. One of the possible solutions of this problem is shown in Figure 5. 
TABLE 1: The results of schematic modeling.

\begin{tabular}{lcccccccc}
\hline & & \multicolumn{3}{c}{ Parameter } & & \\
Scheme & $f_{p}$ & $\delta f_{p}$ & $Q$ & $\delta Q$ & $K_{0}$ & $\delta K_{0}$ & $I_{0}$ & Conditions \\
& $\mathrm{GHz}$ & $\%$ & - & $\%$ & $\mathrm{~dB}$ & $\%$ & $\mathrm{~mA}$ & \\
\hline & 1 & 0.8 & 8.6 & 72 & 13 & 22 & 3 & $C_{1}=294 \mathrm{fF}$ \\
$C_{2}=800 \mathrm{fF}$ \\
$R_{1}=360$ \\
$R_{2}=150$ \\
Figure 4
\end{tabular}

Note: bipolar heterojunction transistors $\left(m=1, l_{e}=840 * 10^{-9}, W_{e}=420 * 10^{-9}, x=8, y=2\right)$ Supply voltage $\pm 2.5 \mathrm{~V}$.

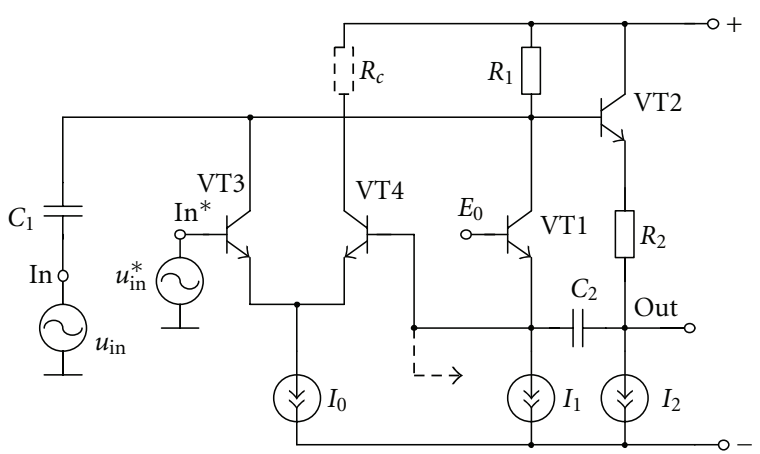

FIGURE 5: Schematic of band-pass section with adjustable pole parameters.

Here

$$
K_{i}=\alpha_{1}+\alpha_{4} \frac{h_{11.1}}{h_{11.3}+h_{11.4}}=\alpha_{1}+\alpha_{4} \frac{I_{0}}{4 I_{1}} .
$$

Thus, by the changing of the mode current $I_{0}$ it is possible at given values of $h_{11.1}$ and $h_{11.2}$ (currents $I_{1}$ and $I_{2}$ ) to change the $K_{i}$ and, therefore, by retaining of any parametric condition (18), (19) (22), (23) to implement the required section parameters. The results of comparative modeling of reviewed schemes in software environment Cadence Virtuoso on the base of technological process SGB25VD components (technological inaccuracy of resistors and capacitors is $\pm 10 \%$ ) are presented in Table 1 .

The increasing of the current amplifier circuit complexity always generates additional structural degrees of freedom, which can be used to implement certain impedance conditions. So in reviewed scheme the input signal source can be connected to the base of VT4, and the load to the emitter of VT1. In this case, at pole parameters retention the gain at the pole frequency is determined by the following relation:

$$
K_{0}=-Q \alpha_{4} \frac{h_{11.1}}{h_{11.3}+h_{11.4}} \sqrt{\frac{\tau_{2}}{\tau_{1}}} \frac{R_{1}}{R_{2}+h_{11.1}+h_{11.2}} .
$$

This property of scheme is convenient to use by additional intersection connections implementation in highorder filters and by integration of band-pass section into different IP-blocks [3].

\section{The Main Conclusions}

In accordance with the stated task of the work and achieved results it is possible to formulate a number of theoretical conclusions. Firstly, the increasing of active elements use efficiency, in particular current amplifiers, is connected with the usage of symmetric frequency-defining chains in their feedback. This property ensures the independence of the pole frequency (5) from the active element gain and also maximal increasing of the $Q$-factor $\left(Q=1 / d_{p}\right)$ (7), (8). Secondly, this property equalizes the impact of the active elements unity gain area $B_{s}$ on the parameters of the poles (10), (11). In particular, the use of noninverting amplifiers $(K>0)$ is associated with the creation of passive frequencydefining chains of band-pass type, at the same time according to (9) the required value of gain is reduced and sufficient structural degrees of freedom for gain area $\mathrm{B}_{s}$ maximization are implemented [9]. Finally, the degree of influence of $B_{s}$ is directly determined by own attenuation of passive circuit pole $D_{p}$. From this point of view the expediency of separated RC circuits using is justified and an area of parametric trade-off between different components of the overall sensitivity of schematics is created. This statement requires some comment.

As follows from the general relation (5) and its consequence (13), the pole frequency is determined mainly by the parameters of resistors and capacitors of passive circuit, hence the maximal deviation of amplitude-frequency characteristic and phase-frequency characteristic of sections is determined by the corresponding sensitivity and technological and temperature variations of resistors $\left(\Theta_{R}\right)$ and capacitors $\left(\Theta_{C}\right)$. That's why this contribution is proportional to the realizable $Q$

$$
\begin{gathered}
{\left[\frac{\Delta|F(j \omega)|}{|F(j \omega)|}\right]_{\max }=Q\left(\Theta_{R}+\Theta_{C}\right),} \\
\Delta \arg F(j \omega)=\left(\Theta_{R}+\Theta_{C}\right) .
\end{gathered}
$$

On the other hand (expressions (9), (14)) the pole attenuation and $Q$-factor are determined by the accuracy of the implementation of these elements ratios $\left(\Delta \Theta_{R}, \Delta \Theta_{C}\right)$, that are significantly less than $\Theta_{R}$ and $\Theta_{C}$ for all technologies. 
Therefore, at the practical problems solution, the realization of higher parametric sensitivity of this parameter may be accepted (almost up to equal the contributions of $\Theta_{R}, \Delta \Theta_{R}$ and $\Theta_{C}, \Delta \Theta_{C}$ ). From (9) and the properties of symmetrical $\mathrm{RC}$ circuits of the second order (the functional dependence of $D_{p}$ and $\left.D_{0}\right)$ follows

$$
\begin{array}{cc}
D_{0}=2 m, & D_{p}=2 m+\frac{1}{m}, \\
S_{m}^{d p}=3 m Q, & S_{K}^{d p}=-Q K \frac{1}{m},
\end{array}
$$

where $m$ is the ratio of single-type parameters of passive elements $(R$ or $C$ ) with accuracy of implementation that is determined by $\Delta \Theta_{R}$ or $\Delta \Theta_{C}$.

Thus, the condition of equality of the contributions is associated with the implementation of conditions

$$
\Theta_{R}=\Delta \Theta_{R} \frac{3 m}{2}, \quad \Theta_{C}=\Delta \Theta_{C} \frac{3 m}{2},
$$

that define acceptable maximal value of

$$
m \leq \frac{2 \Theta_{R}}{3 \Delta \Theta_{R}}, \quad m \leq \frac{2 \Theta_{C}}{3 \Delta \Theta_{C}} .
$$

As can be seen from (30), (10), (11) $m_{\mathrm{opt}}=1$ is the sufficient condition for the nondominant nature of the influence of attenuation on the instability of the frequency characteristics of filters. In this case, $D_{p}=D_{p \min }=3, D_{0}=2$ and, as follows from (9), $K<3$ for any $Q$. So

$$
S_{m}^{d p}=-S_{K}^{d p}=3 Q ; \quad S_{B S}^{\omega p}=S_{B S}^{d p}<-\frac{9}{2} \frac{\omega_{p}}{B_{S}},
$$

and the implementation of the required quality factor is associated with the creation of qualitative current amplifier with $1 \leq K_{i}<3$ [9].

Similarly, for the separated symmetric chains (example in Figure 4) can be obtained

$$
D_{0}=m, \quad D_{p}=m+\frac{1}{m}
$$

and shown that by $m_{\mathrm{opt}}=1$

$$
S_{m}^{d p}=-S_{K}^{d p}=2 Q, \quad S_{B S}^{\omega p}=S_{B S}^{d p}<2 \frac{\omega_{p}}{B_{S}}
$$

and for any $Q$ value it is required

$$
1 \leq K_{i}<2
$$

the lower value of the gain at the wider range of operating frequencies.

The last relation shows the benefits of schemes with separate RC circuits when the specified range of values $K_{i}$ is obtained by bipolar transistors geometry and provides greater stability of the gain.

\section{Conclusion}

The achieved results show that the limiting estimations of the effective usage of active elements for localization of transfer functions poles $\left(\omega_{p}, d_{p}\right)$ of electrical filters create additional opportunities to optimize the structure and parameters of current amplifiers. In this case, as it follows from (35), the optimal relation of parameters of frequency-defining passive elements not only does not increase the overall instability of the frequency characteristics of filters, but also permits to reduce the influence of gain area (unity gain frequency) on the attainable operating frequency range of scheme. That is what ultimately allows to implement the total decomposition of simple procedures in active filters [10]. As seen from reviewed example of band-pass section, by this approach the functional adjustment of their parameters is easily realized via the mode dependence of input impedance and current amplifier gain. The designing of such amplifiers is possible by the use of common microcircuitry principles of self- and mutual compensation of the parasitic parameters influence of both field effect and bipolar transistors.

\section{References}

[1] A. S. Korotkov, D. V. Morozov, and R. Unbehauen, "Lowvoltage continuous-time filter based on a CMOS transconductor with enhanced linearity," International Journal of Electronics and Communications, vol. 56, no. 6, pp. 416-420, 2002.

[2] A. S. Korotkov, D. V. Morozov, H. Hauer, and R. Unbehauen, "A $2.5 \mathrm{~V}, 0.35 \mu \mathrm{m}$ CMOS transconductance-capacitor filter with enhanced linearity," in Proceedings of the 45th Midwest Symposium on Circuits and Systems, vol. 3, pp. III141-III144, Tulsa, Okla, USA, August 2002.

[3] S. G. Krutchinsky, U. I. Ivanov, and I. J. Krikun, "Dynamic range increasing in multichannel polynomial ARC-filters," Radio-engineering, no. 8, 1989.

[4] G. W. Roberts and A. S. Sedra, "All current-mode frequency selective circuits," Electronics Letters, vol. 25, no. 12, pp. 759761, 1989.

[5] A. U. Keskin and E. Hancioglu, "Current mode multifunction filter using two CDBAs," International Journal of Electronics and Communications, vol. 59, no. 8, pp. 495-498, 2005.

[6] E. Yuce and S. Minaei, "Universal current-mode filters and parasitic impedance effects on the filter performances," International Journal of Circuit Theory and Applications, vol. 36, no. 2, pp. 161-171, 2008.

[7] M. Sagbas, U. E. Ayten, and H. Sedef, "Current and voltage transfer function filters using a single active device," IET Circuits Devices and Systems, vol. 4, no. 1, pp. 78-86, 2010.

[8] S. G. Krutchinsky, "Fundamental limitations in precision analog circuits," in Proceeding of the 1st IEEE International Conference on Circuits and Systems for Communications (ICCSC '02), pp. 16-19, St. Peterburg, Russia, 2002.

[9] S. G. Krutchinsky, N. N. Prokopenko, A. S. Budyakov, J. M. Savchenko, and N. V. Kovbasjuk, "Compensation methods of basic transistors output capacitance components in analog integrated circuits," in Proceedings of the European Conference on Circuits and Systems for Communications (ECCSC '06), pp. 44-49, Bucharest, Romania, 2006.

[10] S. G. Krutchinsky, Structural Synthesis in Analogue Microcircuitry, Publishing of SRSUES, Moscow, Russia, 2010. 

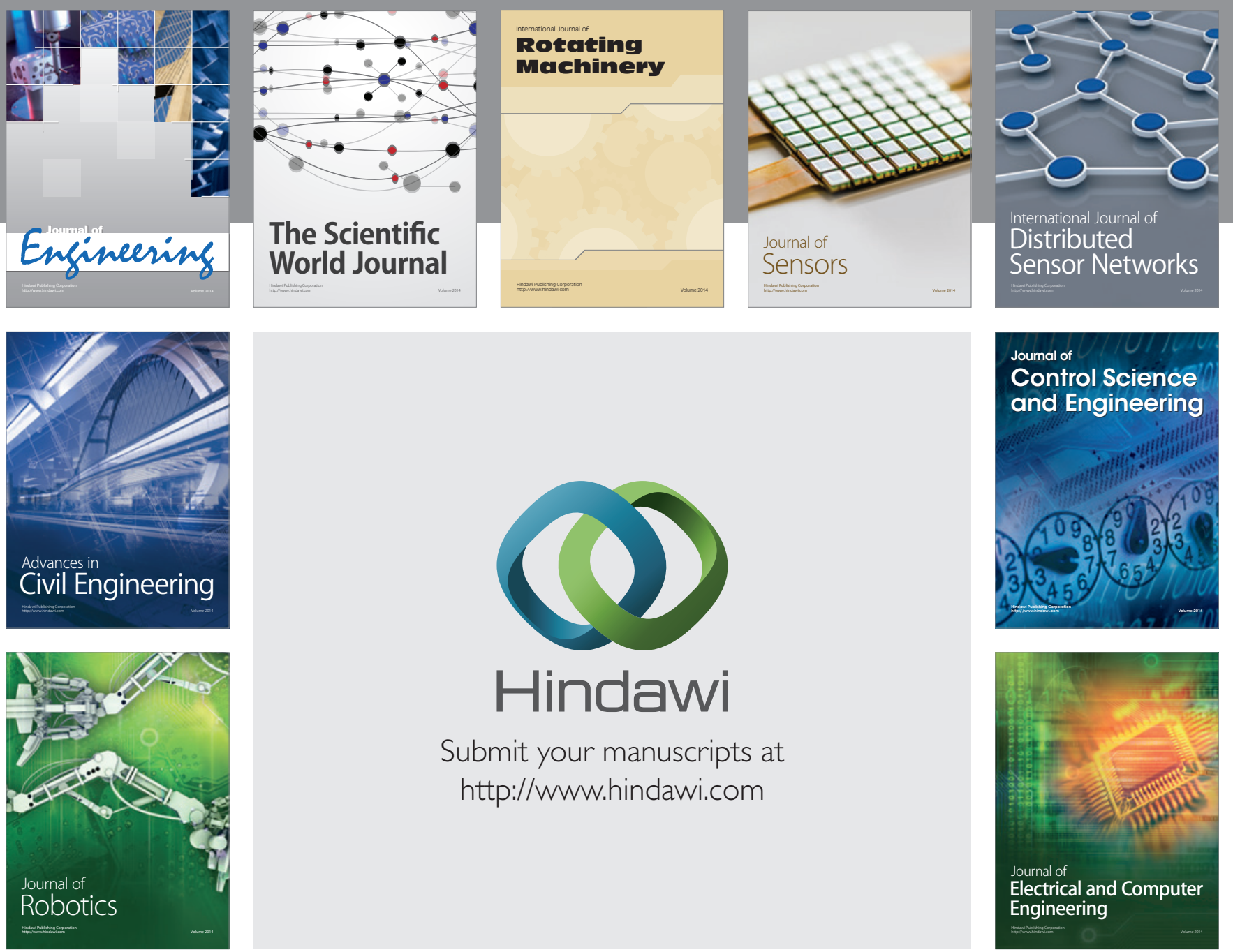

Submit your manuscripts at

http://www.hindawi.com
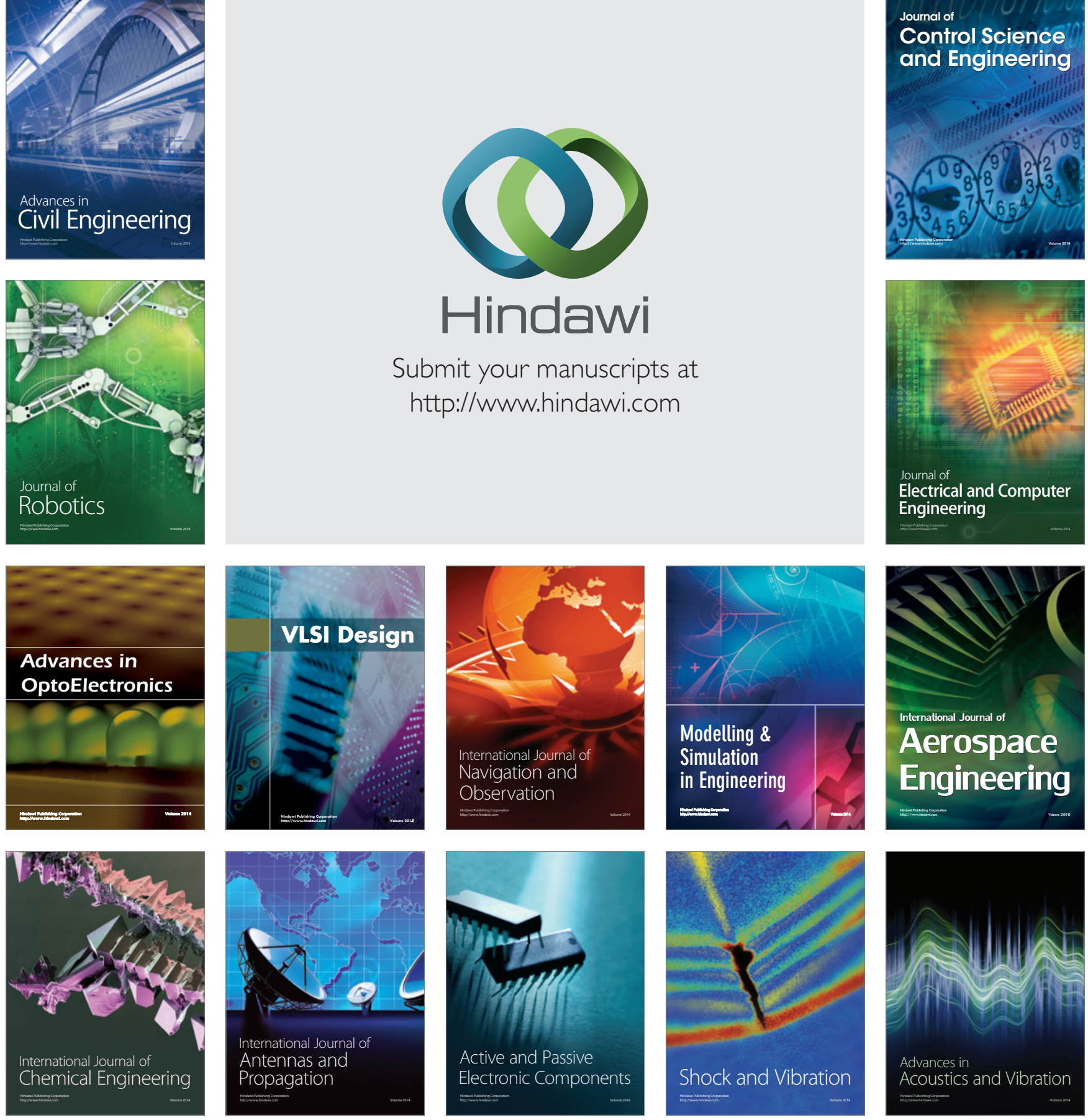